\title{
Simulated altitude exercise training damages small intestinal mucosa barrier in the rats
}

\author{
Meng Li ${ }^{1, \uparrow}$, Tianyu Han ${ }^{2, \dagger}$, Weijia Zhang ${ }^{2}$, Wei Li', Yang Hu², Sang Ki Lee ${ }^{1, *}$ \\ 'Department of Sport Science, Chungnam National University, Daejeon, Korea \\ ${ }^{2}$ Sports Science School, Beijing Sport University, Beijing, China
}

This study investigated the effect of simulated altitude training on the changes of small intestinal mucosa barrier, bacterial overgrowth and inflammatory response in the small intestine of rat. Male 8-week-old Sprague-Dawley rats were randomly divided into four groups: normal oxygen sedentary group $(n=30)$, normal oxygen exercise group $(n=30)$, low oxygen sedentary group $(n=30)$ and low oxygen exercise group $(n=30)$. Exercise training was on a treadmill for $1 \mathrm{hr}$ per day on days 3,6 , and 9 in the hypoxia condition. Hematological profiles, hematolxylin and eosin staining, fluorescence in situ hybridization, reverse transcription-polymerase chain reaction and Western blot were used to analyze the effect of simulated altitude training on the amount of bacteria, and expression of mRNA and protein. Simulated exercise training significantly increased red blood cells and hematocrit. The small intestinal mucosa barrier was significantly injured by the simulated altitude exercise training. Comparatively more bacterial growth was evident in the small intestine by the simulated altitude exercise training. mRNA levels of interleukin-6 (IL-6) and tumor necrosis factor-alpha (TNF- $\alpha$ ) and protein expression of nuclear factor-kappa $B(\mathrm{NF}-\mathrm{\kappa} B$ ) were significantly elevated by simulated altitude exercise training. These results suggest that the simulated altitude exercise training may impair the small intestinal mucosa barrier via elevation of bacterial growth and inflammatory cytokines (IL-6, TNF- $\alpha$ ) and the up-regulation of NF- $\kappa B$ in the rats.

Keywords: Altitude training, Bacteria, Interleukin-6, Small intestinal mucosa barrier, Tumor necrosis factor-alpha, Nuclear factor-kappa B

\section{INTRODUCTION}

High altitude is a special geographic environment that incorporates low pressure and low oxygen levels. The hypoxic environment has been exploited in athletic training in an effort to improve performance (Ashenden et al., 1999; Saunders et al., 2009; Wilber, 2001). The positive effect of altitude training on athletic performance includes increased rate of oxygen utilization, due to increased hemoglobin content and increased oxygen carrying capacity (Pottgiesser et al., 2009; Wachsmuth et al., 2013).

Darkening this picture, several studies reported the association of altitude training with loss of appetite, vomiting, diarrhea, and other gastrointestinal symptoms (Flaherty et al., 2016; Tschöp et al., 1998). Another consequence of prolonged endurance or intense exercise training could be a decrease in the intestinal capacity to avoid infection. Hypoxia conditions may injure the small intestinal antioxidant system and could alter gastrointestinal mucosa microstructure (Deitch, 2002; Maeda et al., 2010; Sun et al., 2004).

Under normal circumstances, the intestinal microecological structure maintains relative balance and stability. With the help of the intestinal mucosa barrier and the immune system, the resident intestinal flora avidly adheres to the intestinal mucosa, resulting in colonization resistance (Garrett et al., 2010). Pathological changes, such as altitude exposure, result in intestinal problems that include derangement of the symbiosis between the host and the normal flora, and imbalance of intestinal microecologies; these changes lead to intestinal mucosa barrier damage (Round and Mazmanian, 2009). The gastrointestinal tract appears ischemic and hypoxic in the earliest stages of stress (Gosain and Gamelli, 2005). Hypoxic ischemia leads to intestinal mucosa epi-
${ }^{*}$ Corresponding author: Sang Ki Lee (iD https://orcid.org/0000-0001-8507-564X Department of Sports Science, Chungnam National University, 99 Daehak-ro, Yuseong-gu, Daejeon 34134, Korea

Tel: +82-42-821-6456, Fax: +82-42-823-0387, E-mail: nicelsk@cnu.ac.kr

${ }^{\dagger}$ These authors contributed equally to this study and should be considered co-first authors. Received: March 1, 2018 / Accepted: April 20, 2018
This is an Open Access article distributed under the terms of the Creative Commons Attribution Non-Commercial License (http://creativecommons.org/licenses/by-nc/4.0/) which permits unrestricted non-commercial use, distribution, and reproduction in any medium, provided the original work is properly cited. 
thelial edema, disrupted mucosal thickness and increased intestinal permeability, which induce endotoxin and bacterial translocation (Nagpal et al., 2006; Yu et al., 2013). Bacteria and endotoxin enter the portal vein or lymphatic system and translocate to other tissues and organs, such as liver, leading to a cascade response mediated by inflammatory mediators, including tumor necrosis factor-alpha (TNF- $\alpha$ ) and interleukin-6 (IL-6), which are downstream factors of nuclear factor-kappa B (NF- kB) (Fan et al., 2015). They play an important role in a variety of inflammatory reactions and are correlated with the severity of inflammation (Ferencz et al., 2006). High altitude hypoxia and intense exercise training independently trigger injury of intestinal mucosa. Therefore, altitude exercise training may cause more severe of intestinal mucosa damage because of the dual simulation of hypoxia and exercise training.

Previous studies have been shown that the altitude hypoxia can damage of intestinal epithelium and increase the levels of inflammatory cytokines. However, the link between altitude training in intestinal mucosa barrier damage and inflammatory responses is unclear. The purpose of this study is to investigate the effect of altitude training on small intestinal barrier damage and levels of TNF- $\alpha$, IL-6, and NF-kB in rats.

\section{MATERIALS AND METHODS}

\section{Animals}

All experiments were performed on 8-week-old male SpragueDawley rats (Vital River Laboratory Animal Technology Co., Ltd, Beijing, China). Rats were housed under controlled temperature $\left(22^{\circ} \mathrm{C}-25^{\circ} \mathrm{C}\right)$, humidity $(75 \%)$ and light conditions (alternating 12-hr light-dark cycle) and were provided food and water ad libitum. The rats were randomly assigned to four groups: normal oxygen sedentary group (NOS, $\mathrm{n}=30$ ), normal oxygen exercise group (NOE, $n=30$ ), low oxygen sedentary group (LOS, $n=30$ ), and low oxygen exercise group (LOE, $n=30$ ). The low oxygen groups were housed in a hypoxic chamber with $12.7 \%$ oxygen to simulate the hypoxic environment at an altitude of $4,000 \mathrm{~m}$. All protocols were approved by the institutional animal care and use committee of Beijing Sport University (2015ZD006).

\section{Exercise training protocol}

A motorized rodent treadmill was used for exercise training. Rats were allowed to adapt to the exercise training under the normal oxygen condition with moderate exercise intensity $(60 \%-$ $70 \%$ maximal oxygen uptake) for 5 days. After that, animals were randomly assigned into normal oxygen exercise and low oxygen exercise group. According to the Bedford protocol (Bedford et al., 1979), animals were trained at $24 \mathrm{~m} / \mathrm{min}, 45 \mathrm{~min} /$ day in moderate intensity on days 3, 6, and 9 until the experimental endpoint.

\section{Tissue isolation}

Rats were sacrificed after the formal exercise training on days 3 , 6 , or 9 . The small intestine was isolated under anesthesia achieved by an intraperitoneal abdominal injection of $3 \%$ pentobarbital ( 30 $\mathrm{mg} / \mathrm{kg}$ ). Blood was collected from the abdominal artery in the presence of an anticoagulation compound and biochemical tests were done within $6 \mathrm{hr}$.

\section{Hematoxylin and eosin staining}

Hematoxylin and eosin staining (H\&E) staining was used to measure small intestine epithelium. Tissues were fixed in $4 \%$ formaldehyde for $24 \mathrm{hr}$ and embedded in paraffin. Serial cross-sections (5- $\mu \mathrm{m}$ thickness) were cut using a model RM 2235 microtome (LEICA, Nussloch, Germany) and were examined under light microscopy using an Eclipse Ni-E microscope (Nikon, Tokyo, Japan). The height and the surface area of small villous and the thickness of mucosa were measured by ImageJ software (National Institute Health, Bethesda, MD, USA). Eight numbers of villous were measured in each $\mathrm{H} \& \mathrm{E}$ section.

\section{Florescence in situ hybridization}

EUB-338 probe (5'-GCTGCCTCCGTAGGAGT-3', $1 \mu \mathrm{g} / \mathrm{mL}$ ) bound to fluorescein isothiocynate (FITC) (BoGoo Biotechnology Co., Ltd., Shanghai, China) was used to detect the bacteria in the small intestine. The FITC has excitation and emission wavelengths of 494 and $518 \mathrm{~nm}$, respectively, and emits green fluorescence. The probe binds to a conserved region of bacterial $16 \mathrm{~S}$ rRNA that is specific for Eubacteria. Tissue sections were counterstained with $5 \mathrm{mg} / \mathrm{mL}$ 4',6-diamidino-2-phenylindole (DAPI; Biotime, Beijing, China) to visualize eukaryotic cell nuclei. Imaging was performed using a LSM710 confocal microscope (Carl Zeiss, Jena, Germany) at $\times 200$ magnification with image data analyzed using Image pro Plus image software.

\section{RT-PCT}

Total RNA was isolated from intestine using RNA isolation Kit (Tiangen Biotech Co., Ltd., Beijing, China). Reverse transcription of mRNA was achieved using an ImProm-II Reverse Transcription System (Promega, Madison, WI, USA). The levels of beta-actin RNA were used as endogenous controls for normal- 
ization rat RNA. The relative gene expressions were calculated using cycle threshold $(\mathrm{Ct}$ ) values in accordance with the $\Delta \mathrm{Ct}$ method. Primer sequences were as follows: Beta-actin, forward: 5'-CCCATCTATGAGGGTTACGC-3', backward: 5’ TTTAATGTCACGCACGATTTC-3’; TNF- $\alpha$, forward: 5'-GAGATGTGGAACTGGCAGAGGA-3'; backward: 5'-TCAGTAGACAGAAGAGCGTGGTG-3'; and IL-6, forward: 5'-CCTACCCCAACTTCCAATGCT-3', backward: 5'-GGTCTTGGTCCTTAGCCACT-3’.

\section{Western blot}

Small intestine tissues were homogenized in lysis buffer (50 $\mathrm{mM}$ Tris and $\mathrm{pH} 7.4,150 \mathrm{mM} \mathrm{NaCl}, 1 \%$ Triton X-100, $1 \%$ sodium deoxycholate, $0.1 \%$ sodium dodecyl sulfate (SDS), $2 \mathrm{mM}$ sodium pyrophosphate, $25 \mathrm{mM} \beta$-glycerophosphate, $1 \mathrm{mM}$ ethylenediaminetetraacetic acid, $1 \mathrm{mM} \mathrm{Na} 3 \mathrm{VO} 4,0.5-\mu \mathrm{g} / \mathrm{mL}$ leupeptin; Solarbio Science \& Technology Co., Ltd. Beijing, China) and centrifuged for $5 \mathrm{~min}$ at $12,000 \mathrm{rpm}$ and $4{ }^{\circ} \mathrm{C}$. The supernatant was removed and protein in the pellet was quantified using a BCA assay Kit (Solarbio Science \& Technology Co., Ltd.). Proteins (30 $\mu \mathrm{g})$ were resolved using 12\% SDS-polyacrylamide gel electrophoresis and transferred to a polyvinylidene fluoride membrane. Nonspecific reaction of the membrane was removed by blocking it for $1 \mathrm{hr}$ on ice in $5 \%$ bovine serum albumin (BSA) in Tris-buffered saline (TBS). NF-кB (Cell Signaling Technology, Waltham, MA, USA) and $\beta$-actin (Cell Signaling Technology) were incubated overnight at $4{ }^{\circ} \mathrm{C}$ in TBS with $3 \%$ BSA. Horseradish peroxidase-labeled secondary antibody (Biotime) was diluted 1:5,000 and added. Blots were developed for visualization using an enhanced chemiluminescence detection kit (Thermo Fisher, San Diego, CA, USA). GIS software (Tanon, Shanghai, China) was used to quantify expression.

\section{Statistical analyses}

Values are expressed as mean \pm standard error of the mean. The statistical evaluation was conducted with a one-way analysis of variance and two-way repeated measured analysis of variance of variance followed by a Duncan's post hoc test, and $P<0.05$ was considered statistically significant.

\section{RESULTS}

\section{Effect of simulated altitude training on body weight}

In order to investigate the effect of simulated altitude exercise training on body weight, we've analyzed the changes of body

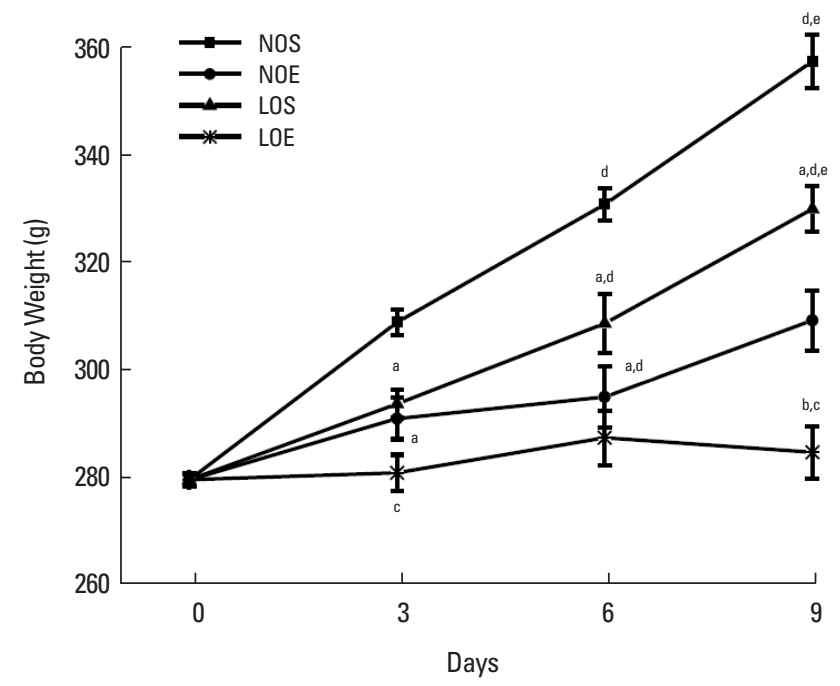

Fig. 1. Simulated altitude training inhibited the elevation of body weight in rats, both under the normal oxygen and low oxygen conditions. NOS, normal oxygen with sedentary; NOE, normal oxygen with exercise training; LOS, low oxygen with sedentary; LOE, low oxygen with exercise training. Each data indicated mean \pm standard error of the mean $(n=10)$. ${ }^{a} P<0.05$ vs. NOS. ${ }^{b} P<0.05$ vs. NOE. ${ }^{\mathrm{c}} P<0.05$ vs. LOS. ${ }^{\mathrm{d}} P<0.05$ vs. day $3 .{ }^{e} P<0.05$ vs. day 6 .

weight in normal and low oxygen conditions (Fig. 1). Significantly restrained persistent increasing of body weight under the normal oxygen and low oxygen conditions compared with sedentary groups ( $P<0.05$, NOS vs. NOE; LOS vs. LOE, Fig. 1$)$. There was significantly greater inhibition of body weight increase in the simulated exercise training group compared with the normal oxygen exercise group.

\section{Effect of simulated altitude training on hematological profiles}

In whole blood, red blood cells (RBCs) and the percentage of hematocrit (HCT) were significantly enhanced in LOS and LOE groups compared with NOS and NOE groups $(P<0.05)$ (Fig. 2). Also the low oxygen exercise training group significantly increased RBC number but decreased the percentage of HCT compared with low oxygen sedentary at day 6 , respectively $(P<0.05$, LOS vs. LOE). Considering training time as an influential factor, RBC and HCT were compared at different times. No temporal relationship was apparent for RBC but was suggested for HCT. The NOE and NOS groups were similar.

\section{Effect of simulated altitude training on intestinal mucosa} barrier

We compared the small intestine in the NOS, NOE, LOS, and LOE groups at different times (Fig. 3). Microscopic examinations 

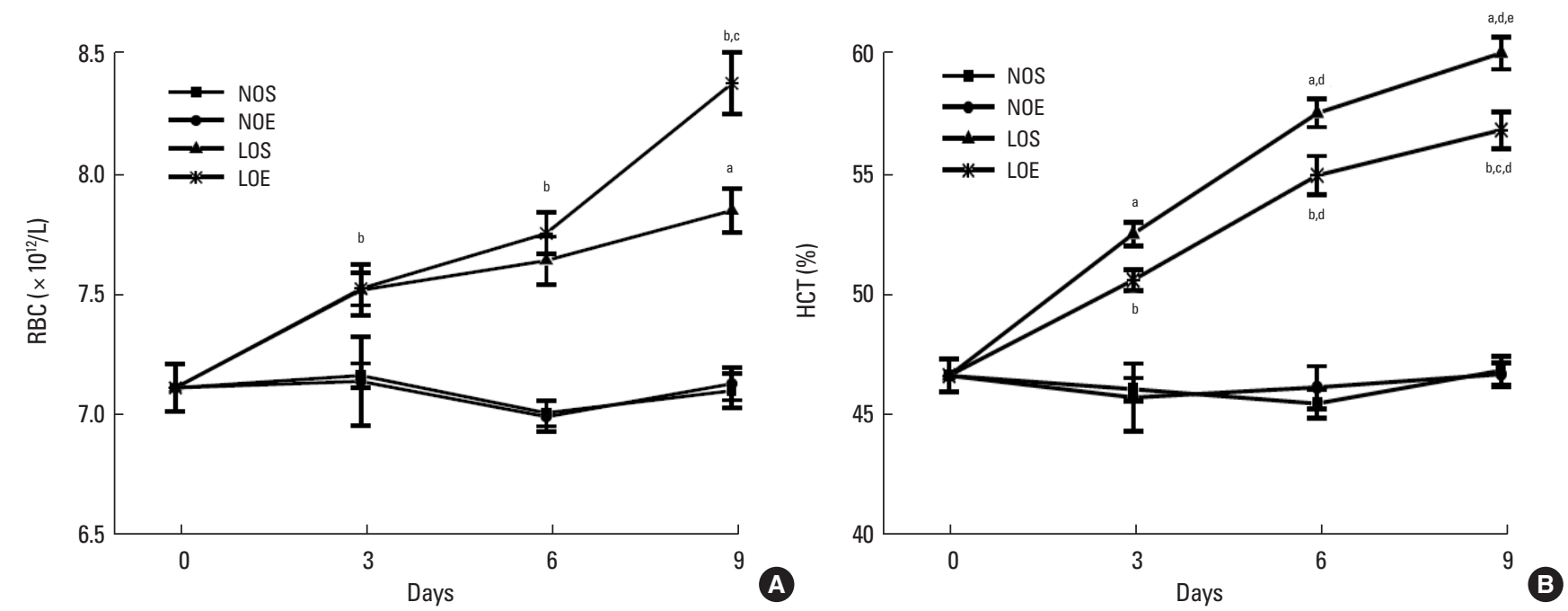

Fig. 2. Simulated altitude training enhanced the elevation of haematological parameters: (A) red blood cells (RBCs), (B) hematocrit (HCT). NOS, normal oxygen with sedentary; NOE, normal oxygen with exercise training; LOS, low oxygen with sedentary; LOE, low oxygen with exercise training. Each data indicated mean \pm standard error of the mean $(n=10)$. ${ }^{a} P<0.05$ vs. NOS. ${ }^{b} P<0.05$ vs. NOE. ${ }^{c} P<0.05$ vs. LOS. ${ }^{d} P<0.05$ vs. day $3 .{ }^{e} P<0.05$ vs. day 6 .

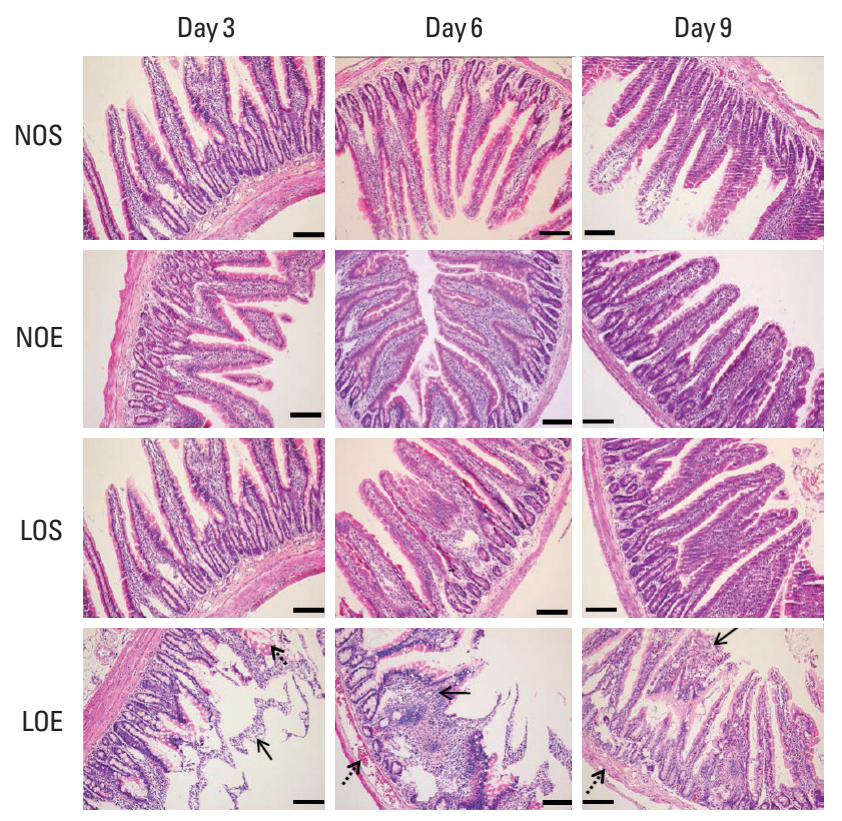

Fig. 3. Simulated altitude training damaged small intestinal mucosa barrier. NOS, normal oxygen with sedentary; NOE, normal oxygen with exercise training; LOS, low oxygen with sedentary; LOE, low oxygen with exercise training. Solid arrows indicate villi swelling and peeling from submucosa; dotted lines indicate congestion. Scale bar is $100 \mu \mathrm{m}$.

revealed pathological changes in the villi. Obviously swelling of villi were evident in tissue obtained from the LOE group at days 3, 6, and $9(P<0.05)$ (Fig. 3). H\&E staining additionally revealed peeling of tissue from the submucosa. Congestion was evident in different locations including submucosa and the periphery of villi.
The most severe damage of the intestinal mucosal barrier was evident at day 6 in the LOE group.

\section{Effect of simulated altitude training on bacteria in the small intestine}

Florescence in situ hybridization analysis shows that EUB-338 positive bacteria were significantly increased in LOS and LOE groups compared with NOS and NOE groups $(P<0.05)$ (Fig. 4) and EUB-338 positive bacteria of LOE group was significantly increased compared with LOS group at the same times $(P<0.05)$ (Fig. 4).

\section{Effect of simulated altitude training on mRNA levels of TNF-a and IL-6}

IL- 6 and TNF- $\alpha$ mRNA levels of small intestine in the LOE group were significantly increased compared with the NOE and LOS groups, respectively $(P<0.05)$ (Fig. 5). Significant time-dependent changes of the levels of IL- 6 and TNF- $\alpha$ levels were evident in the LOS and LOE groups.

\section{Effect of simulated altitude training on small intestine NF-KB}

NK- $\mathrm{KB}$ expression was significantly increased in the LOS and LOE group compared with the NOS and NOE groups at the same times, respectively $(P<0.05)$ (Fig. 6). Significantly increasing NF- $\mathrm{KB}$ was evident in the LOE group on day 6 , but statistical significance was not evident at days 3 and 9 . 

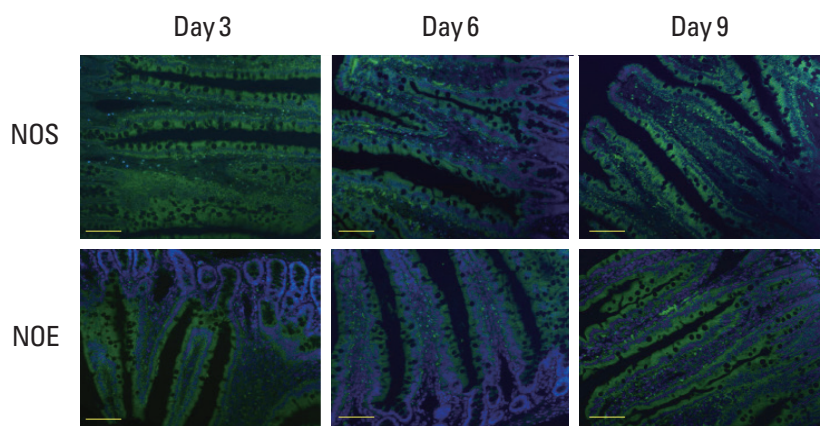

LOS
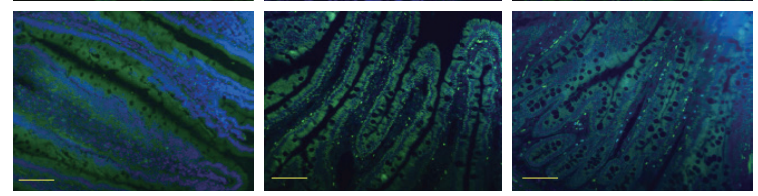

LOE
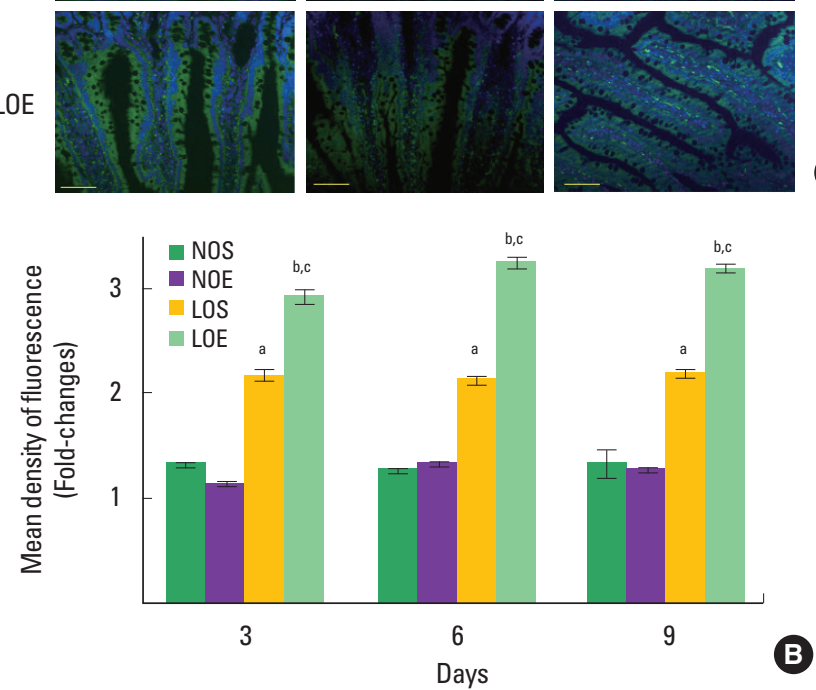

Fig. 4. Simulated altitude training increased the amount of bacteria in small intestine. (A) Fluorescence in situ hybridization presented and located bacteria, (B) mean density of 5'-GCTGCCTCCGTAGGAGT-3' (EUB-338) probe positive fluorescence. Each data indicate mean \pm standard error of the mean $(n=10)$. NOS, normal oxygen with sedentary; NOE, normal oxygen with exercise training; LOS, low oxygen with sedentary; LOE, low oxygen with exercise training. DAPI (4',6-diamidino-2-phenylindole), blue; EUB-338 probe, green. ${ }^{a} P<0.05$ vs. NOS. ${ }^{\mathrm{b}} P<0.05$ vs. NOE. ${ }^{\mathrm{c}} P<0.05$ vs. LOS.

\section{DISCUSSION}

There are three major findings. Simulated altitude training damaged the small intestinal mucosa barrier, increased the amount of bacteria in small intestine and elevated the levels of IL- 6 and TNF- $\alpha$ due to the up-regulation of NF- $\kappa B$ in the small intestine.

In general, people tend to display symptoms of hypoxia at altitudes exceeding 2,000 $\mathrm{m}$, with symptoms being more severe in those who experience a greater change in altitude, such as from
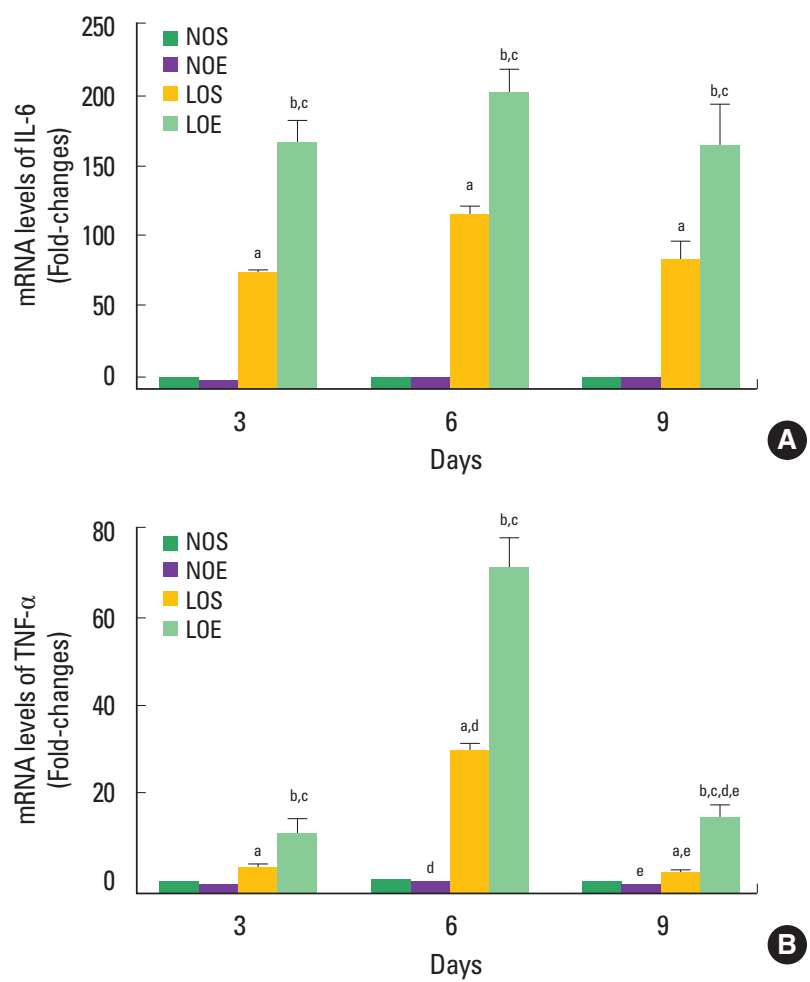

Fig. 5. Simulated altitude training increased mRNA levels of interleukin-6 (IL-6) and tumor necrosis factor-alpha (TNF- $\alpha$ ) in small intestine. (A) Change of mRNA levels of IL-6, (B) change of mRNA levels of TNF- $\alpha$. Each data indicate mean \pm standard error of the mean $(n=10)$. NOS, normal oxygen with sedentary; NOE, normal oxygen with exercise training; LOS, low oxygen with sedentary; LOE, low oxygen with exercise training. ${ }^{a} P<0.05$ vs. NOS. ${ }^{b} P<0.05$ vs. NOE. ${ }^{~} P<0.05$ vs. LOS. ${ }^{\mathrm{d}} P<0.05$ vs. day $3 .{ }^{\mathrm{e}} P<0.05$ vs. day 6 .

sea level. RBC and HCT are used to evaluate the effect of altitude training adaptation in athletes or animal models. The variations of these blood factors are involved in hypoxia and altitude training. Most often, hypoxia or altitude conditions will result in increased RBC and HCT (Bor-Kucukatay et al., 2014 ; Stokke et al., 1986). The present results from simulated altitude training reaffirm the view that a low oxygen environment boosts RBC and HCT.

In the early days after exposure to high altitude, about half of all people will experience nausea, vomiting, diarrhea and other gastrointestinal symptoms, collectively termed "high altitude gastrointestinal stress syndrome" (Anand et al., 2006). This syndrome is closely related with hypoxia-induced intestinal mucosa barrier dysfunction. Hypoxic exposure is a physiological stimulus; its biological impact on the body mainly depends on the length and intensity of hypoxia exposure. Acute or persistent hypoxic exposure causes intestinal mucosa injury more easily (Kannan et al., 2011). Recavarren-Arce et al. (2005) examined gastric biopsy samples and described more serious gastrointestinal mucosa injuries 

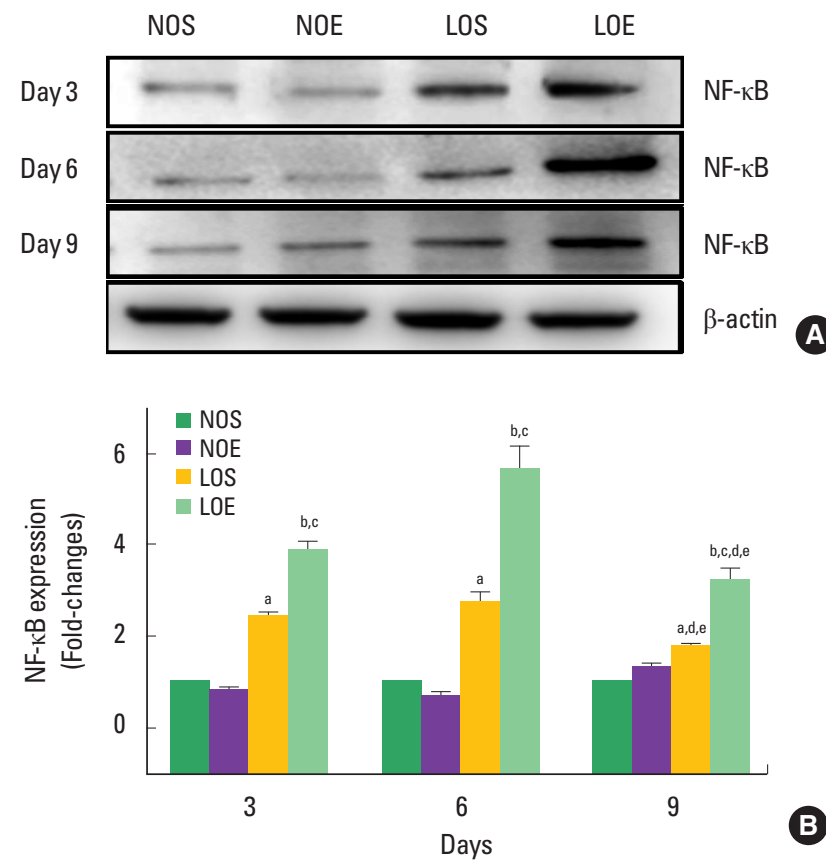

Fig. 6. Simulated altitude training increased protein expression of nuclear factor-kappa B (NF-kB) expression in small intestine. (A) Western blot showed NF$\kappa B$. (B) Fold-changes of NF- $\kappa B$ for each group. Each data indicate mean \pm standard error of the mean $(n=10)$. NOS, normal oxygen with sedentary; NOE, normal oxygen with exercise training; LOS, low oxygen with sedentary; LOE, low oxygen with exercise training. ${ }^{\mathrm{a}} P<0.05$ vs. NOS. ${ }^{\mathrm{b}} P<0.05$ vs. NOE. ${ }^{\circ} P<0.05$ vs. LOS. ${ }^{\mathrm{d}} P<0.05$ vs. day 3 . ${ }^{\mathrm{e}} P<0.05$ vs. day 6 .

in Peruvians dwelling in the Andes at an altitude of $3,700 \mathrm{~m}$ compared with individuals living at sea level through gastric biopsy. Zhang et al. (2015) indicated the link between morphological changes of the small intestine after rapid entry to high altitudes; villi swelling and hyperemia of small intestinal lamina propia with visible inflammatory granulocytes were evident.

The normal intestinal barrier include a mechanical barrier of intestinal mucosa epithelium, immune barrier, chemical barrier and biological barrier. Our research mainly focused on the effect of simulated altitude training on the mechanical intestinal mucosa barrier. H\&E staining revealed swelled small intestinal villi and shortened villi length in the LOE group on days 6 and 9, respectively. Microscopic observation revealed mild pathological changes at day 9 compared with day 6 in the LOE group. These results suggest hypoxia resistance among the LOE rats depending on the duration of hypoxia exposure.

The intestinal mucosa barrier comprises epithelial cells and tight junction. The latter guards against bacterial invasion into intestinal lamina propria, blocks the gap between adjacent epithelial cells and maintains the stability of the intestinal mucosal bar- rier function to avoid abnormal mucosal immune response (Groschwitz and Hogan, 2009). When the body encounters stress, the gastrointestinal tract reacts first to ischemia and hypoxia (Secchi et al., 2000). Thus, the gastrointestinal tract is more prone to injury, such as intestinal mucosa atrophy. On the other hand, the intestinal tract immunity organ is more sensitive to physical or environmental stress. Compared with the sea level environment, altitude hypoxia exposure is more excessive. This will diminish intestinal tract function and promote translocation of invading pathogenic bacteria and endotoxin into blood and other organs through the damaged intestinal mucosa barrier (Boros et al., 1995; Pedersen and Hoffman-Goetz, 2000; Rowell, 1983).

The endotoxin and bacteria induce intestinal endotoxemia. Endotoxin translocation is more common than bacterial translocation. Endotoxin translocation leads to a vicious cycle that aggravates intestinal mucosa barrier damage (Wang et al., 2004). Once the intestinal barrier is breached, damage can be caused by invading pathogens or from overgrowth of endogenous pathogens, such as bacteria. Invasive bacteria can damage the mucosal layer and intestinal epithelial cells as well as the lamina propria (Zeitouni et al., 2016). This process can be considered a negative-interactive feedback. We used the EUB338 probe to detect bacteria in the small intestine. The results indicated that hypoxia and altitude training induced an imbalance in the flora of the small intestine. The number of microorganisms was highest at day 3 in the LOS and LOE groups.

High altitude hypoxia injure the intestinal mucosal barrier includes swelling and decreased length of villi in rats (Zhang et al., 2015). These findings provide a cautionary note for athletes engaged in high altitude training. Another study examined the expression levels of Toll-like receptor (TLR)/NF- $\kappa B$, IL-6, and TNF- $\alpha$, and the ultrastructure of the small intestinal mucosa in a rat model of hypoxia (Luo et al., 2012). These findings indicate that hypoxia can induce increased expression of NF- $\mathrm{B}$ mRNA and protein. The authors concluded that hypoxia-induced small intestinal barrier damage and bacterial translocation might underlie the TLR/NF- $\kappa \mathrm{B}$ signaling pathway. In the present study, we detected the expression of NF- $\mathrm{kB}$ protein and mRNA levels of TNF- $\alpha$ and IL-6. All were sharply increased by low oxygen and simulated altitude training. Interestingly, NF- $\kappa \mathrm{B}$ expression was suppressed in the NOE group compared with the NOS group.

As a transcription factor, NF- $\mathrm{kB}$ stimulates the activity of a variety of inflammatory cytokines and induces the copious release of inflammatory factors. These mediators induce apoptosis of intestinal epithelial cells and injury of intestinal tract tissue, and have 
been implicated with organ dysfunction. TNF- $\alpha$ and IL- 6 are pivotal inflammatory factors located downstream of NF- $\mathrm{kB}$. They play an important role in multiple inflammatory reactions and are closely related to the severity of inflammation (Spehlmann and Eckmann, 2009).

IL-6 is another inflammatory mediator and induced by NF- $\mathrm{kB}$ activation. Also, NF- $\mathrm{kB}$ is critical for TNF- $\alpha$-induced inflammatory reactions. The function of the intestinal barrier may be regulated by inflammatory cytokines such as TNF- $\alpha$ and IL-6 (Topol and Kamyshny, 2013). In particular, they can accelerate the inflammatory reaction of intestinal organs under hypoxic state that mediate the inflammatory response and damage the function of the intestinal barrier.

Altitude training is widely used by professional athletes to improve performance. The present results add to the concern of the potential damage of this pursuit on inflammatory damage of the gastrointestinal tract. The specific signaling pathway of NF- $\mathrm{kB}$ and the possibility of bacterial or endotoxin translocation through the breached intestinal mucosal barrier should be investigated.

Taken together, these data suggest that the simulated altitude exercise training may impair the small intestinal mucosa barrier, which allows increasing of growth of bacteria by elevation of IL-6, TNF- $\alpha$, and NF- $\kappa B$ in intestine of rats.

\section{CONFLICT OF INTEREST}

No potential conflict of interest relevant to this article was reported.

\section{ACKNOWLEDGMENTS}

This work was supported by the Ministry of Education of the Republic of Korea and the National Research Foundation of Korea (NRF-2017S1A5A2A01026677).

\section{REFERENCES}

Anand AC, Sashindran VK, Mohan L. Gastrointestinal problems at high altitude. Trop Gastroenterol 2006;27:147-153.

Ashenden MJ, Gore CJ, Martin DT, Dobson GP, Hahn AG. Effects of a 12day "live high, train low" camp on reticulocyte production and haemoglobin mass in elite female road cyclists. Eur J Appl Physiol Occup Physiol 1999;80:472-478.

Bedford TG, Tipton CM, Wilson NC, Oppliger RA, Gisolfi CV. Maximum oxygen consumption of rats and its changes with various experimen- tal procedures. J Appl Physiol Respir Environ Exerc Physiol 1979;47: 1278-1283.

Bor-Kucukatay M, Colak R, Erken G, Kilic-Toprak E, Kucukatay V. Altitude training induced alterations in erythrocyte rheological properties: a controlled comparison study in rats. Clin Hemorheol Microcirc 2014;58:479-488.

Boros M, Takaichi S, Hatanaka K. Ischemic time-dependent microvascular changes and reperfusion injury in the rat small intestine. J Surg Res 1995;59:311-320.

Deitch EA. Bacterial translocation or lymphatic drainage of toxic products from the gut: what is important in human beings? Surgery 2002;131: 241-244.

Fan Z, Yao J, Li Y, Hu X, Shao H, Tian X. Anti-inflammatory and antioxidant effects of curcumin on acute lung injury in a rodent model of intestinal ischemia reperfusion by inhibiting the pathway of NF-Kb. Int J Clin Exp Pathol 2015;8:3451-3459.

Ferencz A, Racz B, Gasz B, Benko L, Jancso G, Kurthy M, Roth E. Intestinal ischemic preconditioning in rats and NF-kappaB activation. Microsurgery 2006;26:54-57.

Flaherty G, O'Connor R, Johnston N. Altitude training for elite endurance athletes: a review for the travel medicine practitioner. Travel Med Infect Dis 2016;14:200-211.

Garrett WS, Gordon JI, Glimcher LH. Homeostasis and inflammation in the intestine. Cell 2010;140:859-870.

Gosain A, Gamelli RL. Role of the gastrointestinal tract in burn sepsis. J Burn Care Rehabil 2005;26:85-91.

Groschwitz KR, Hogan SP. Intestinal barrier function: molecular regulation and disease pathogenesis. J Allergy Clin Immunol 2009;124:3-20.

Kannan KB, Colorado I, Reino D, Palange D, Lu Q, Qin X, Abungu B, Watkins A, Caputo FJ, Xu DZ, Semenza GL, Deitch EA, Feinman R. Hypoxia-inducible factor plays a gut-injurious role in intestinal ischemia reperfusion injury. Am J Physiol Gastrointest Liver Physiol 2011; 300:G853-861.

Luo H, Guo P, Zhou Q. Role of TLR4/NF-kB in damage to intestinal mucosa barrier function and bacterial translocation in rats exposed to hypoxia. PLoS One 2012;7:e46291.

Maeda T, Miyazono Y, Ito K, Hamada K, Sekine S, Horie T. Oxidative stress and enhanced paracellular permeability in the small intestine of methotrexate-treated rats. Cancer Chemother Pharmacol 2010;65: $1117-1123$

Nagpal K, Minocha VR, Agrawal V, Kapur S. Evaluation of intestinal mucosal permeability function in patients with acute pancreatitis. Am J Surg 2006;192:24-28.

Pedersen BK, Hoffman-Goetz L. Exercise and the immune system: regulation, integration, and adaptation. Physiol Rev 2000;80:1055-1081. 
Pottgiesser T, Ahlgrim C, Ruthardt S, Dickhuth HH, Schumacher YO. Hemoglobin mass after 21 days of conventional altitude training at 1816 m. J Sci Med Sport 2009;12:673-675.

Recavarren-Arce S, Ramirez-Ramos A, Gilman RH, Chinga-Alayo E, Watanabe-Yamamoto J, Rodriguez-Ulloa C, Miyagui J, Passaro DJ, Eza D. Severe gastritis in the Peruvian Andes. Histopathology 2005; 46:374-379.

Round JL, Mazmanian SK. The gut microbiota shapes intestinal immune responses during health and disease. Nat Rev Immunol 2009;9:313323.

Rowell LB. Cardiovascular aspects of human thermoregulation. Circ Res 1983;52:367-379.

Saunders PU, Pyne DB, Gore CJ. Endurance training at altitude. High Alt Med Biol 2009;10:135-148.

Secchi A, Ortanderl JM, Schmidt W, Gebhard MM, Martin E, Schmidt H. Effect of endotoxemia on hepatic portal and sinusoidal blood flow in rats. J Surg Res 2000;89:26-30.

Spehlmann ME, Eckmann L. Nuclear factor-kappa B in intestinal protection and destruction. Curr Opin Gastroenterol 2009;25:92-99.

Stokke KT, Rootwelt K, Wergeland R, Vale JR. Changes in plasma and red cell volumes during exposure to high altitude. Scand J Clin Lab Invest Suppl 1986;184:113-117.

Sun J, Wang XD, Liu H, Xu JG. Ketamine suppresses intestinal NF-kappa $\mathrm{B}$ activation and proinflammatory cytokine in endotoxic rats. World J Gastroenterol 2004;10:1028-1031.

Topol I, Kamyshny A. Study of expression of TLR2, TLR4 and transckription factor NF-kB structures of galt of rats in the conditions of the chronic social stress and modulation of structure of intestinal microflora. Georgian Med News 2013;(225):115-122.

Tschöp M, Strasburger CJ, Hartmann G, Biollaz J, Bärtsch P. Raised leptin concentrations at high altitude associated with loss of appetite. Lancet 1998;352:1119-1120.

Wachsmuth NB, Völzke C, Prommer N, Schmidt-Trucksäss A, Frese F, Spahl O, Eastwood A, Stray-Gundersen J, Schmidt W. The effects of classic altitude training on hemoglobin mass in swimmers. Eur J Appl Physiol 2013;113:1199-1211.

Wang ZT, Yao YM, Xiao GX, Sheng ZY. Risk factors of development of gut-derived bacterial translocation in thermally injured rats. World J Gastroenterol 2004;10:1619-1624.

Wilber RL. Current trends in altitude training. Sports Med 2001;31:249265.

Yu J, Liu F, Yin P, Zhao H, Luan W, Hou X, Zhong Y, Jia D, Zan J, Ma W, Shu B, Xu J. Involvement of oxidative stress and mitogen-activated protein kinase signaling pathways in heat stress-induced injury in the rat small intestine. Stress 2013;16:99-113.

Zeitouni NE, Chotikatum S, von Köckritz-Blickwede M, Naim HY. The impact of hypoxia on intestinal epithelial cell functions: consequences for invasion by bacterial pathogens. Mol Cell Pediatr 2016;3:14.

Zhang F, Wu W, Deng Z, Zheng X, Zhang J, Deng S, Chen J, Ma Q, Wang Y, Yu X, Kang S, Wang X. High altitude increases the expression of hypoxia-inducible factor $1 \alpha$ and inducible nitric oxide synthase with intest-inal mucosal barrier failure in rats. Int J Clin Exp Pathol 2015; 8:5189-5195. 